Témoigner Témoigner. Entre histoire et mémoire

Getuigen Revue pluridisciplinaire de la Fondation Auschwitz

122 | 2016

Révisionisme et négationisme

\title{
Le roman graphique Irmina : un entretien sur la discrimination, les choix personnels et la mémoire
}

Een vraaggesprek over discriminatie, keuzevrijheid en herinnering

Fransiska Louwagie et Fabian Van Samang

Traducteur : Michel Teller

\section{(Q) OpenEdition}

Journals

Édition électronique

URL : https://journals.openedition.org/temoigner/4108

DOI : 10.4000/temoigner.4108

ISSN : 2506-6390

Cet article est une traduction de :

Een vraaggesprek over discriminatie, keuzevrijheid en herinnering - URL : https://

journals.openedition.org/temoigner/4123 [nl]

Éditeur :

Éditions du Centre d'études et de documentation Mémoire d'Auschwitz, Éditions Kimé

Édition imprimée

Date de publication : 2 mai 2016

Pagination : 46-51

ISSN : 2031-4183

Référence électronique

Fransiska Louwagie et Fabian Van Samang, « Le roman graphique Irmina : un entretien sur

la discrimination, les choix personnels et la mémoire », Témoigner. Entre histoire et mémoire [En ligne],

122 | 2016, mis en ligne le 30 septembre 2021, consulté le 03 février 2022. URL : http://

journals.openedition.org/temoigner/4108; DOI : https://doi.org/10.4000/temoigner.4108 
En 2014, Barbara Yelin a publié Irmina un roman graphique racontant l'histoire d'une jeune Allemande qui s'engage dans le national-socialisme. Nous nous sommes entretenus avec l'auteure et avec l'historien Alexander Korb, qui a contribué à l'œuvre en qualité de conseiller scientifique et qui a également signé la postface.

$\rightarrow$ Entretien mené par

Fransiska Louwagie - Lecturer in French Studies, Stanley Burton Centre for Holocaust and Genocide Studies, University of Leicester

Fabian Van Samang - Docteur en histoire et enseignant au Klein Seminarie de Roeselare

Traduit du néerlandais par Michel Teller

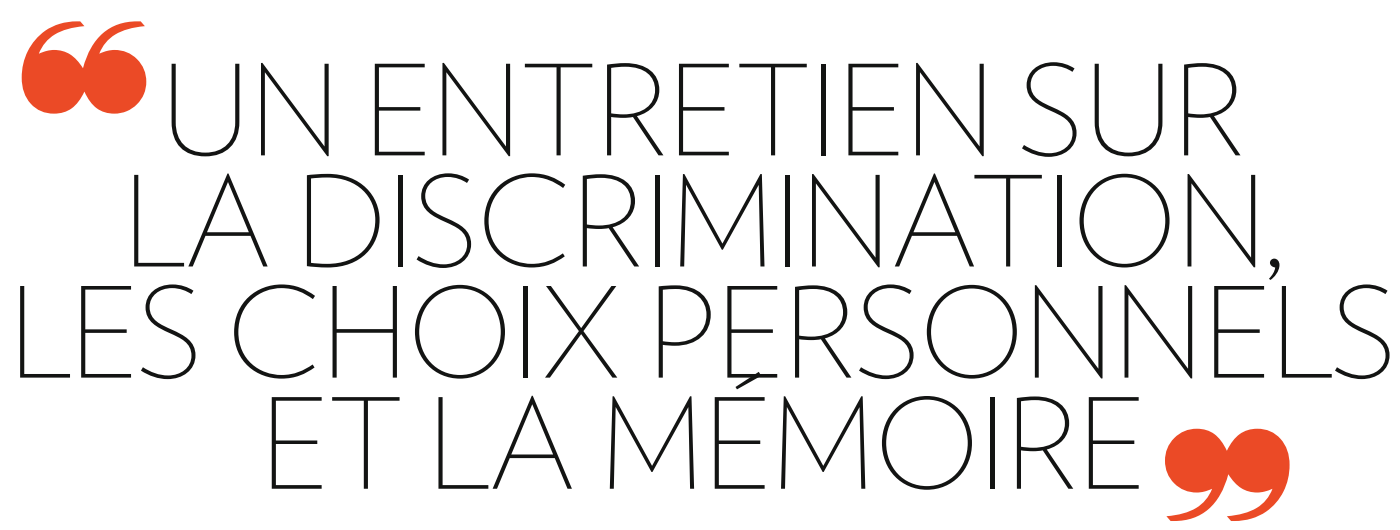

Irmina raconte l'histoire d'une jeune fille allemande qui voulait mener sa propre vie, mais qui, à la suite de circonstances, d'un manque d'initiative et peut-être de considérations pragmatiques, devient vecteur d'un discours nazi auquel elle avait d'abord été assez indifférente. Cette exploration de "ce qui a mal tourné » est-elle une quête personnelle de sens et peut-être aussi une question incontournable pour la «troisième génération » en Allemagne? Dans quelle mesure les questions posées dans le roman graphique diffèrent-elles de celles qui ont été mises en avant par les précédentes générations?

Barbara Yelin : Pendant que je travaillais à ce livre, j’ai parlé à des Allemands de ma génération et je me suis aperçue que la façon dont la plupart d'entre eux abordaient l'histoire de leur famille était très similaire à la mienne : on apprend beaucoup de choses sur l'histoire du national-socialisme et de la Shoah, on s'en souvient, on y réfléchit, mais souvent on ne s'intéresse pas de près au rôle que des membres de notre famille, qu'il s'agisse de nos grands-parents ou de leurs propres parents, ont joué à cette époque. C'est une question importante pour à peu près tous ceux à qui j’ai parlé.
Il y a beaucoup de « vides mémoriels » dans les souvenirs des familles et les sentiments de culpabilité qui accompagnent ceux-ci, comme l'a dit Harald Welzer dans son livre Opa war kein Nazi. Des vides qui, souvent, ne peuvent plus être comblés aujourd'hui parce que cette génération a en grande partie disparu.

Je ne sais pas si nous posons un autre type de questions que la dernière génération, celle de nos parents. Mais je suis certaine que de nombreuses questions n’ont pas été posées à ce jour.

Alexander Korb : Je voudrais abonder dans le sens de Barbara. Il est désormais admis que les Allemands, dans leur ensemble, ont reconnu leur responsabilité dans les événements de la Shoah et que leur exemple montre comment une nation peut gérer l'héritage d'un génocide. En même temps, exporter la Shoah pour en faire un symbole du génocide dans le monde présente aussi un risque. Si nous la qualifions de crime suprême, ily a un risque qu'elle passe à un niveau très abstrait, où certains responsables sont assimilés au mal par excellence alors que, sur le terrain, dans la vie des Allemands ordinaires, la Shoah a souvent pris des formes d'exclu- •.• 


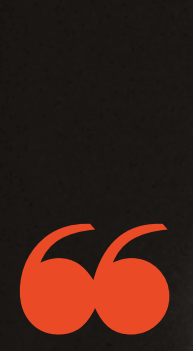

Bar qra Yelin a fait d s étud d'art ê | Université de

I . a travaillé en utualité I'U V r ssité de Saarb iicken eld nime des ateliers pour Institut Goetho diautres associalions culturelles.
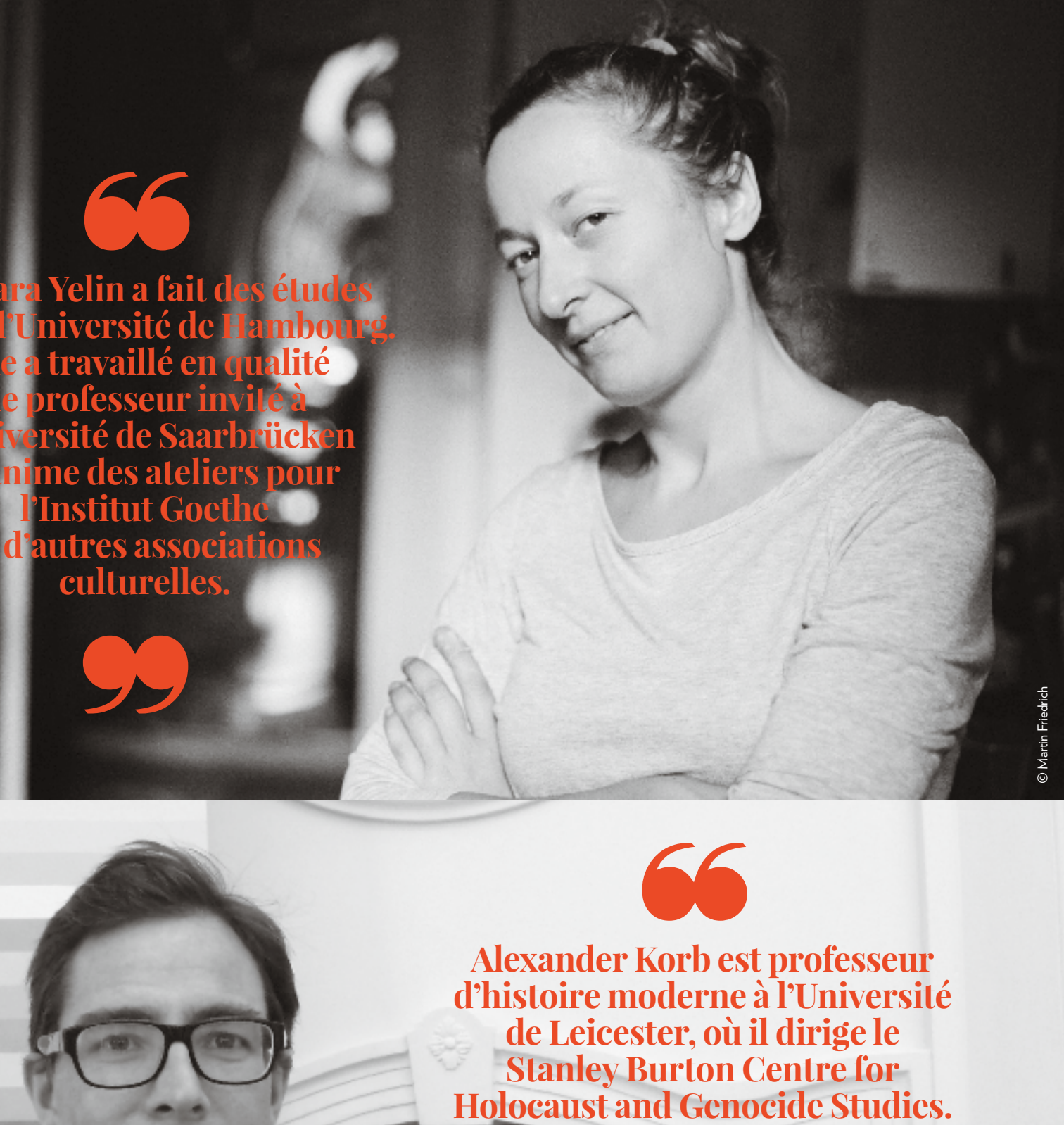

Alexander Korb est professeur d'histoire moderne à l'Université de Leicester, où il dirige le Stanley Burton Centre for Holocaust and Genocide Studies.

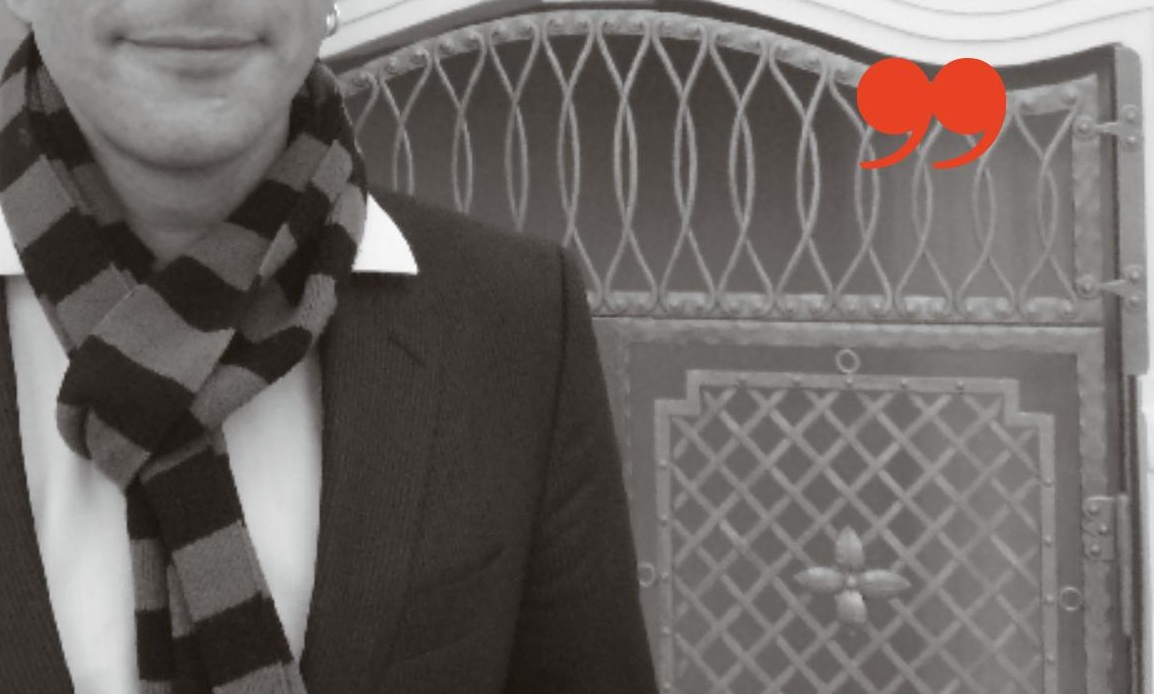


•. sion simples et quotidiennes. Ce n'est pas neuf: quand on lit notamment des témoignages de survivants, on en apprend beaucoup sur les modes de discrimination banals et mesquins dont les Juifs ont fait l'objet. Mais Barbara le montre du point de vue d'une Allemande ordinaire. Je trouve cette perspective tout à fait remarquable : elle nous tend un miroir.

Le roman dépeint une jeune femme qui s'oppose au racisme quand celui-ci vise son ami noir Howard, à Londres, dans les années trente, mais qui adhère au nazisme (ou, à tout le moins, qui ferme les yeux sur lui) dans les années quarante. Ceci semble lié à un changement plus large d'état d'esprit et d'attitude, y compris visà-vis d'elle-même et de son pays. Comment l'interaction complexe et souvent personnelle des intérêts d'Irmina illustre-t-elle plus largement le contexte allemand et peutêtre même les réactions sélectives aux discriminations en général?

BY : Au début, dans l'Angleterre de 1935, Irmina se sent elle-même marginalisée. Pas seulement comme Allemande à l'étranger, que l'on considère bien sûr avec scepticisme à cette époque, mais aussi comme une femme de la classe moyenne qui ne veut pas se marier et qui veut gagner sa vie. Quand elle se lie d'amitié avec Howard, qui souffre de discriminations et de racisme, elle se bat pour lui. Elle veut être à ses côtés et, s’il est discriminé, elle se sent également humiliée, parce qu'elle se sent d'une certaine façon liée à lui. Mais un peu plus tard, Irmina devient elle-même raciste parce qu'elle est jalouse de la réussite d'Howard à Oxford. Ce qui a toujours compté le plus pour elle, c'est son propre statut et ses ambitions. Comme Howard le lui a dit le jour où elle a pris sa défense : «Tu ne l'as pas fait pour moi, tu l'as fait pour toi. »

Comme elle ne veut pas se préoccuper de politique ou prendre position contre l'Allemagne nazie, même en vivant en Angleterre, il n'y a pas chez elle de véritable revirement quand elle part pour l'Allemagne. En fin de compte, la contradiction n'est qu'apparente. De retour en Allemagne, où Gregor la pousse à rejoindre le groupe toujours plus nombreux et plus puissant des partisans et des bénéficiaires du nazisme, elle renonce à son projet de mener une vie indépendante et elle épouse cet architecte nazi, membre des SS, conformément à sa volonté d'ascension sociale. Elle ne voit et elle ne condamne la discrimination que quand elle en est ellemême victime. C'est une vision très nombriliste.
AK : Je voudrais ajouter qu'Irmina est aussi caractéristique des millions d'Allemands qui n'étaient ni des électeurs ni des partisans nazis avant 1933, mais qui ont été de plus en plus impressionnés par leurs succès, réels et prétendus, et par les opportunités que cela leur offrait. Pour cela, il fallait fermer les yeux sur la persécution des Juifs et d'autres minorités, et c'est un prix que la plupart des Allemands ont accepté de payer.

Plusieurs romanciers, dont l'écrivain français André Schwarz-Bart, ont mis en rapport la persécution des Juifs et l'esclavage ou la discrimination et le racisme envers les Noirs. Dans le roman graphique, au contraire, nous avons des histoires contrastées entre l'émancipation réussie d'un citoyen britannique noir et le manque d'émancipation d'une femme allemande, qui n'a pas su mener à bien les " grands projets » qu'elle avait conçus pour elle-même. Cette opposition dénonce-t-elle l'absence de réflexion critique dans les milieux allemands de l'époque ou viset-elle spécifiquement le rôle et l'attitude des femmes? Le roman graphique cherche-t-il aussi à faire comprendre la situation d'Irmina ? Quelle est la fonction de la réussite de son ami noir dans cette perspective ? Et le contraste relativement net entre les deux histoires poursuit-il un objectif (éducatif) particulier?

BY : En fait, oui et non. Comme je le mentionne dans la préface, le contraste entre les deux parcours a été un fait - un fait qui a suscité une question en moi : comment suivre les projets que l'on a dans la vie et quelles sont les conséquences ? Bien sûr, il serait difficile d'imaginer qu'Howard renonce à ses ambitieux projets parce qu'une jeune femme blanche lui brise le cœur à vingt ans. Cela n'aurait pas fonctionné, ni dans la réalité, ni comme intrigue dans mon roman.

Cela démontre que leurs attitudes sont aussi typiques de ce qu'ils représentent - Irmina comme femme allemande blanche, Howard comme étudiant noir originaire des Caraïbes - et des opportunités qu'ils ont pu avoir ou non. La dimension féminine la plus marquée est qu'Irmina avait toujours la possibilité de se replier sur sa vie privée en trouvant un mari alors que ce n'est pas imaginable pour Howard.

AK : Le roman de Barbara est une belle démonstration de ce qu'on appelle l'intersectionnalité, l'étude des intersections entre des formes ou des systèmes d'oppression, de domination ou de discrimination. Nombreux sont ceux qui ont décrit en ces termes la rivalité 


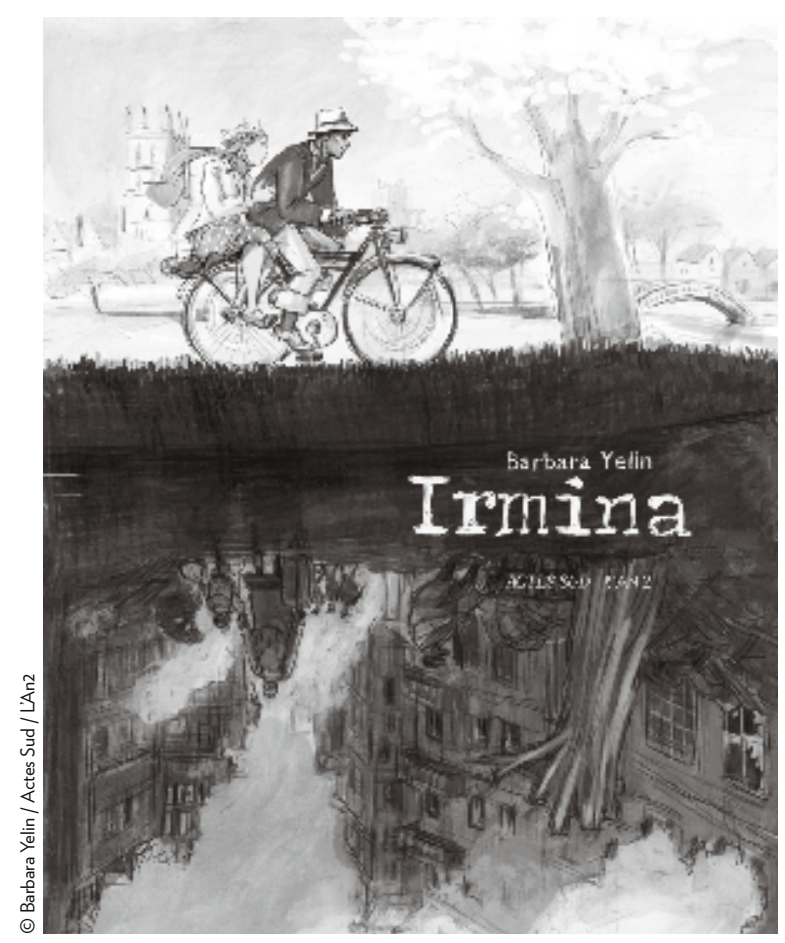

- Couverture du roman graphique Irmina (Actes Sud BD, 288 pages, traduit de l'allemand par Paul Derouet, ISBN 978-2-330-03641-6)

entre Barack Obama et Hillary Clinton lors des primaires démocrates en 2008 et qui en ont conclu prématurément que «la race l'emporte sur le genre.» Howard est discriminé en raison de son origine raciale, mais il peut poursuivre une carrière masculine. En tant que femme, Irmina est confrontée à des discriminations, mais elle bénéficie dans une large mesure de sa qualité d'Allemande blanche. Ce n'est pas une coïncidence s'ils tombent amoureux l'un de l'autre à Londres, où ils sont tous les deux des marginaux. L'histoire n'est pas imaginable à Berlin, où Irmina n'était pas une marginale ; et on ne peut pas attendre non plus que son histoire d'amour avec un homme noir la rende critique vis-àvis de la persécution des Juifs dans l'Allemagne nazie.

Il est un fait que, sous le régime nazi, les femmes allemandes ont pu faire des choix et qu'elles en ont activement fait usage, très souvent aux dépens de groupes qui étaient discriminés, comme nous l'ont appris des études récentes sur les crimes nazis perpétrés par des femmes. Le repli sur la vie privée était une autre option possible, comme Barbara le suggère. À cet égard, d'ailleurs, Irmina n'est pas une victime, c'est son choix de s'engager dans cette voie.

Les sources auxquelles vous puisez ont leur origine tantôt dans votre histoire familiale, celle de votre grandmère en particulier, tantôt dans d'autres documents. Comment le travail de création s'est-il déroulé ?

BY : C'est comme un puzzle. J'ai combiné de nombreux éléments de mon histoire familiale avec des éléments d'autres existences comparables ou simplement avec des choses imaginables. Mon but n'était pas en soi de faire la biographie de ma grand-mère.

J'ai été intéressée par les questions qui se posaient après avoir trouvé des journaux personnels et des lettres provenant de sa succession. Je voulais réaliser un roman en images et j’ai expressément modifié certaines parties de son histoire pour les besoins du scénario et pour me concentrer sur les aspects qui m’intéressaient. Avec l'aide d’Alexander,j'ai situé cette histoire familiale dans le contexte plus large de l'ère nazie. Par exemple, les scènes où Irmina passe dans l'espace public, assiste à des émeutes antisémites ou se joint à des manifestations du Parti sont souvent empruntées à des photographies de l'époque. Certains lecteurs les reconnaîtront.

Au-delà de cela, comment la collaboration avec un historien fonctionne-t-elle en pratique et quel a été son impact sur l'élaboration du roman graphique ? À quelle phase du travail cette interaction a-t-elle eu lieu et comment percevez-vous l'un et l'autre le processus et le produit fini ? Y a-t-il eu des différences d'approche et d'interprétation?

BY : J'ai fait pas mal de recherches moi-même et je suis très reconnaissante à Alexander pour le travail de vérification qu'il a effectué. Mais ce qui a été encore plus important pour moi, ce sont nos conversations sur la façon dont l'histoire d'Irmina pouvait refléter des questions générales sur le refus de voir les crimes des nazis : la dissimulation et le silence qui ont été pratiqués au sein même des plus petites entités, les familles qui ne voulaient pas voir ce qui se passait.

AK : Pour moi, cela a été une opportunitéétonnante. Il est rare qu'un historien aitl'occasion de contribueràla réalisation d'un roman graphique. J'ai été extrêmement ••• 


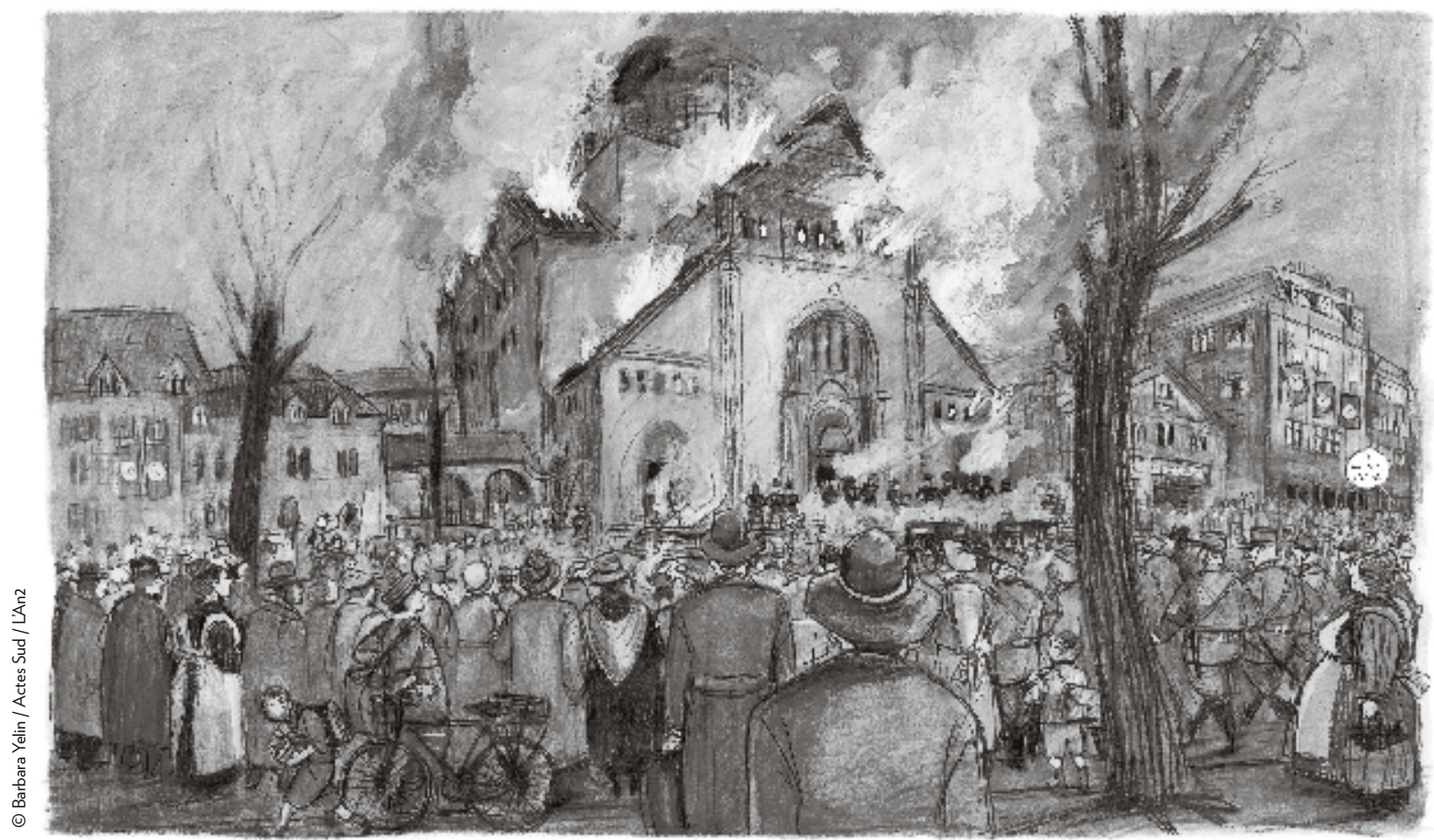

•• impressionné par la profondeur des recherches que Barbara avait menées pour ce livre et par le sentiment qu'elle en avait retiré. Même si elle n’a pas lu tous les débats sur la responsabilité des Allemands ordinaires ou sur le puissant attrait exercé par la Volksgemeinschaft nazie sur les femmes allemandes, elle avait tout compris et elle a raconté un récit qui est à la hauteur des débats que les historiens ont aujourd'hui. Mafonction était donc plutôt de la rassurer par rapport à ce qu'elle faisait.

Mais la réflexion en sens inverse est aussi intéressante :je me suis mis à réfléchir à mon propre travail et cela m’a rendu un peu jaloux de ne pas pouvoir utiliser des personnages fictifs. Peut-être le ferai-je un jour?

Alors que le roman graphique est centré sur des relations de complicité, il ne contient pas de références directes au génocide : l'accent est plutôt mis sur les épreuves quotidiennes de la population allemande, ainsi que sur ses luttes ou ses différences idéologiques. Cela étant, quel rapport le roman entretient-il avec la question de la connaissance ou de l'ignorance du peuple allemand à cette époque?

BY : C'est ce que j'ai précisément voulu savoir en effectuant mes recherches et en construisant mon récit : qu'est-ce qu'une personne comme Irmina pouvait savoir à propos de la Shoah? En fait, il y a plusieurs occasions où elle aurait pu en savoir plus, mais elle refuse d'entendre ce que des gens lui disent. Elle aurait pu savoir, mais elle évite de le faire. Elle se concentre sur sa vie quotidienne et elle se retire de plus en plus derrière les rideaux de son salon.

AK : Je trouve qu'Irmina est un magnifique exemple qui illustre la thèse selon laquelle ne pas savoir n'était pas une coïncidence, mais un choix actif. Décider de ne pas savoir a demandé del'énergie, de la conscience, unebonne part d'ignorance active et une capacité à se tromper soimême. Et cela a provoqué un extrême manque d'empathie, parce que cela obligeait à détourner constamment le regard de ce qui aurait pu interpeller la conscience. De nombreux Allemands ont développé de bonnes compétences pour «ne pas savoir», ce qui les a aidés à jouer les victimes après la guerre. La nécessité de se protéger de la vérité démontre bien sûr aussi à quel point la connaissance du génocide était omniprésente en Allemagne.

Dans le même ordre d'idées, le mari haut placé d'Irmina semble impliqué dans la politique gouvernemen- 
tale, mais il ne parle guère des atrocités, des tueries, de l'horreur. Après avoir été longtemps un tabou dans la représentation de la Shoah, est-ce à présent devenu une référence culturelle partagée (et peut-être évidente) qui fait partie des connaissances encyclopédiques du lecteur? Comment voyez-vous tous les deux le rôle des références et des informations historiques dans le roman graphique, par rapport à la Shoah elle-même, mais aussi plus largement au contexte de l'époque?

BY : Je suppose bien entendu que la réalité historique de l'Allemagne nazie et de la Shoah est connue du lecteur. Le livre ne fonctionne que dans le contexte d'une histoire connue, les choses qui n'ont pas été vues par les protagonistes. Mais la postface d'Alexander est très importante pour moi et est plus qu'un appendice, elle fait partie intégrante du livre. Le roman montre la perception d'Irmina et soulève des questions que la postface situe, si nécessaire, dans leur contexte historique.

AK : C'est une question intéressante, car moi-même, je ne sais pas toujours quel est l'état des connaissances des étudiants dans ma classe. La Shoah fait office de référence culturelle très large et tout le monde semble savoir exactement ce qu'il faut entendre par là. Mais dès le moment où je commence à poser des questions à mes étudiants, il apparaît que leur conception de la Shoah peut en fait être très vague.

En ce qui concerne le roman, je pense qu'il constitue un bon équilibre entre l'information et la fiction, et, de plus, Barbara parvient précisément à évoquer l'indicible, le silence et la mauvaise conscience parmi les Allemands de l'époque. Pour les historiens, il semble beaucoup plus difficile d'évoquer ces sentiments. Le livre peut être utilisé à l'école, mais ce n'est pas un manuel scolaire et si on avait donné davantage « d'informations contextuelles », cela aurait pu nuire au scénario. J'ai écrit une brève postface qui montre les liens entre le roman et les débats historiques actuels sur les attitudes du peuple allemand durant la Shoah.

Dès la couverture, la couleur a manifestement une fonction symbolique dans le roman. Comment l'utilisation de la couleur et de la forme reflète-t-elle l'époque et l'évolution de l'histoire?

BY : La couleur et le format des cases ont été un instrument de soutien au scénario. Au début, ily a davan- tage d'espace, les couleurs sont plus vives, le trait plus libre, ce qui reflète les possibilités et les visions plus larges des protagonistes. Cela évolue vers des tonalités plus sombres et un découpage de la planche en cases plus étroites dans la seconde partie à Berlin pour montrer que la vision d'Irmina se rétrécit elle aussi. Dans la troisième partie, qui se déroule dans les années 1980, on retrouve de la lumière et de nouvelles couleurs pour intensifier l'impression d'un changement d'époque.

Certains romans graphiques ont joué un rôle clé pour raviver la mémoire de la Shoah ou d'autres cas de génocides et d'exterminations massives. Votre œuvre a-t-elle subi des influences particulières et comment définiriezvous le potentiel du médium dans ce domaine?

BY : Bien entendu, Maus n'a pas seulement été très important pour moi et pour mon œuvre, mais aussi pour l'évolution du roman graphique en général, c'est d'ailleurs sans doute l'œuvre la plus importante. Mais j'ai aussi été influencée par d'autres romans, films, ouvrages théoriques et œuvres d'art moderne. Je ne dirais pas que la bande dessinée se prête mieux à la réflexion et à l'évocation de ces sujets, mais je pense qu'elle peut le faire aussi bien. Le dessin offre évidemment des possibilités très particulières. La théorie de la BD nous apprend que voir ou « lire» des visages simplement esquissés ou des têtes d'animaux - comme dans Maus - facilite l'identification avec les personnages et les individualise pour le lecteur. Raconter une histoire en la dessinant a quelque chose de très spécial.

Comment voyez-vous la signification et l'importance de votre roman graphique aujourd'hui pour les jeunes, les étudiants et les lecteurs en général ? Quelles sont les questions que vous espérez soulever?

BY : Irmina est avant tout un roman et non un livre d'histoire. Et j'espère qu'il sera lu par des lecteurs de tout âge. Pour moi, il était plus important de poser des questions que de donner des réponses. Je pense que le livre peut soulever des questions qui ne sont pas seulement liées à l'histoire, mais qui sont éternelles : faut-il être fidèle à ses convictions? Prendre position? Comparer l'évolution de la vie et ses croyances antérieures? Détourner le regard parce que c'est plus confortable? Dans toute leur complexité, ces questions offrent des sujets qui, je l'espère, pourront pousser les lecteurs à réfléchir par eux-mêmes. 